\title{
Using Artificial Neural-Networks in Stochastic Differential Equations Based Software Reliability Growth Modeling
}

\author{
Sunil Kumar Khatri ${ }^{1}$, Prakriti Trivedi ${ }^{2}$, Shiv Kant ${ }^{2}$, Nisha Dembla ${ }^{3}$ \\ ${ }^{1}$ Amity Institute of Information Technology, Amity University, Noida, India; ${ }^{2}$ Government Engineering College, Ajmer, India; \\ ${ }^{3}$ Department of CSE \& IT, NGF College of Engineering \& Technology, Palwal, India. \\ Email: sunilkkhatri@gmail.com
}

Received July $4^{\text {th }}$, 2011; revised July 31 ${ }^{\text {st }}, 2011$; accepted September $8^{\text {th }}, 2011$.

\begin{abstract}
Due to high cost of fixing failures, safety concerns, and legal liabilities, organizations need to produce software that is highly reliable. Software reliability growth models have been developed by software developers in tracking and measuring the growth of reliability. Most of the Software Reliability Growth Models, which have been proposed, treat the event of software fault detection in the testing and operational phase as a counting process. Moreover, if the size of software system is large, the number of software faults detected during the testing phase becomes large, and the change of the number of faults which are detected and removed through debugging activities becomes sufficiently small compared with the initial fault content at the beginning of the testing phase. Therefore in such a situation, we can model the software fault detection process as a stochastic process with a continuous state space. Recently, Artificial Neural Networks $(A N N)$ have been applied in software reliability growth prediction. In this paper, we propose an ANN based software reliability growth model based on Ito type of stochastic differential equation. The model has been validated, evaluated and compared with other existing NHPP model by applying it on actual failure/fault removal data sets cited from real software development projects. The proposed model integrated with the concept of stochastic differential equation performs comparatively better than the existing NHPP based model.
\end{abstract}

Keywords: Software Reliability Growth Model, Artificial Neural Network, Stochastic Differential Equation (SDE), Stochastic Process

\section{Introduction}

\subsection{Software Reliability Growth Modeling}

There are numerous instances where failures of computercontrolled systems have led to colossal loss of human lives and money. With increased complexity of products design, shortened development cycles and highly destructive consequences of software failures, a major responsibility lies in the areas of Software Debugging, Testing and Verification. As software systems have become more and more complex, the importance of effective, well planned testing has increased many folds.

The Software Reliability Growth Model (SRGM) is a tool, which can be used to evaluate the software reliability, develop test status, schedule status and monitor the changes in reliability performance. Several Software Reliability models have been discussed in the literature.
Most of these are based upon historical failure data collected during the testing phase. These models have been utilized to evaluate the quality of the software and for future reliability predictions. Software reliability engineering (SRE) addresses all these issues, from design to testing to maintenance phases.

The Software Reliability Growth Model (SRGM) is a tool of SRE that can be used to evaluate the software quantitatively, develop test status, schedule status and monitor the changes in reliability performance [1,2]. In the last two decades several Software Reliability models have been developed in the literature showing that the relationship between the testing time and the corresponding number of faults removed is either Exponential or S-Shaped or a mix of the two [1,2]. The software includes different types of faults and each fault requires different strategies and different amounts of testing effort 
to remove it.

A number of faults are detected and removed during the long testing period before the system is released to the market. However; the users then find number of faults and the software company then release an updated version of the system. Thus in this case the number of faults that remain in the system can be considered to be a stochastic process with continuous state space [3]. Yamada et al. [4] proposed a simple software reliability growth model to describe the fault detection process during the testing phase by applying Ito type Stochastic Differential Equation (SDE) and obtain several software reliability measures using the probability distribution of the stochastic process. Later on they proposed a flexible Stochastic Differential Equation Model describing a faultdetection process during the system-testing phase of the distributed development environment [4]. Lee et al. [5] used SDE to represent a per-fault detection rate that incorporate an irregular fluctuation instead of an NHPP, and consider a per-fault detection rate that depends on the testing time $t$.

\subsection{Artificial Neural Networks}

Many papers are published in the literature addressing that neural networks offer promising approaches to software reliability estimation and prediction. Karunanithi et al. [6-8] first applied neural network architecture to estimates the software reliability. They also illustrated the usefulness of connectionist models for software reliability growth predictions. Cai et al. [9] used the recent 50 inter-failure times as the multiple-delayed-inputs to predict the next failure time and found the effect of the number of input neurons, the number of neurons in the hidden layer and the number of hidden layers by independently varying the network architecture. They advocated the development of fuzzy software reliability growth models in place of probabilistic software reliability models.

Sherer [10] has applied neural networks for predicting software faults in several NASA projects. Khoshgoftar et al. [11] used the neural network as a tool for predicting the number of faults in a program and concluded that the neural networks produce models with better quality of fit and predictive quality.

$\mathrm{Su}$ et al. [12] have proposed a neural network based approach to software reliability assessment combining various existing models into a Dynamic Weighted Combinational Model (DWCM). Kapur et al. [13] have proposed an ANN based Dynamic Integrated Model (DIM), which is an improvement over DWCM given by Su et al. [12]. Kapur et al. [14] have proposed a Generalized Dynamic Integrated Model (GDIM) using ANN approach, which incorporates the concept of $n$ types of faults. Kha- tri et al. [15] have proposed an artificial neural-network based SRGM considering two types of imperfect debugging during fault removal phenomenon. They considered that during a removal attempt a fault might be removed imperfectly. Such a situation results in number of failures being more than number of removals and is known as imperfect fault debugging. Otherwise it may happen that a fault is generated while removing some fault and existence of a generated fault is known only after the perfect removal of original fault. Due to error generation the total fault content increases.

The paper is organized as follows. Section 2 presents the model formulation for the proposed model. Model is validated in Sections 3 and 4 based on two data sets cited in the literature. Section 5 concludes the paper.

\section{Framework for Modeling}

\subsection{Notations for the Proposed SRGM Using SDE}

$(N(t))$ : The number of faults detected during the testing time $t$ and is a random variable;

$E(N(t))$ : Expected number of faults detected in the time interval $(0, \mathrm{t}]$ during testing phase;

$a$ : Total fault content;

$b$ : Fault detection rates for simple, hard and complex faults;

$\sigma$ : Positive constant that represents the magnitude of the irregular fluctuations for faults;

$\gamma(t)$ : Standardized Gaussian White Noise for faults.

\subsection{Assumptions for the Proposed SRGM Using SDE}

1) The Software fault-detection process is modeled as a stochastic process with a continuous state space.

2) The number of faults remaining in the software system gradually decreases as the testing procedure goes on.

3) Software is subject to failures during execution caused by faults remaining in the software.

4) During the fault isolation/removal, no new fault is introduced into the system and the faults are debugged perfectly.

\subsection{Framework for Modeling for Proposed SRGM}

Several SRGM are based on the assumption of NHPP, treating the fault detection process during the testing phase as a discrete counting process. Recently Yamada et al. [16] observed that if the size of the software system is large, the number of software faults detected during the testing phase becomes large and the change of the number of faults which are detected and removed through debugging activities becomes sufficiently small com- 
pared with the initial fault content at the beginning of the testing phase. So, in order to describe the stochastic behavior of the fault detection process, we can use a Stochastic Model with continuous state space. Since the latent faults in the software system are detected and eliminated during the testing phase, the number of faults remaining in the software system gradually decreases as the testing progresses. Therefore, it is reasonable to assume the following differential equation

$$
\frac{\mathrm{d} N(t)}{\mathrm{d} t}=r(t)[a-N(t)]
$$

where $r(t)$ is a fault-detection rate per remaining fault at testing time $\mathrm{t}$.

However, the behavior of $r(t)$ is not completely known since it is subject to random effects such as the testing effort expenditure, the skill level of the testers, the testing tools and so on and thus might have irregular fluctuation. Thus, we have

$$
r(t)=b(t)+\text { noise }
$$

Let $\gamma(t)$ be a standard Gaussian white noise and $\sigma$ a positive constant representing a magnitude of the irregular fluctuations. So Equation (2) can be written as

$$
r(t)=b(t)+\sigma \gamma(t)
$$

Hence, Equation (1) becomes [17]

$$
\frac{\mathrm{d} N(t)}{\mathrm{d} t}=[b(t)+\sigma \gamma(t)][a-N(t)]
$$

Equation (4) can be extended to the following stochastic differential equation of an Ito Type [3 and 16]

$$
\begin{aligned}
\mathrm{d} N(t)= & {\left[b(t)-\frac{1}{2} \sigma^{2}\right][a-N(t)] \mathrm{d} t } \\
& +\sigma[a-N(t)] \mathrm{d} w(t)
\end{aligned}
$$

where $W(t)$ is a one-dimensional Wiener process, which is formally defined as an integration of the white noise $\gamma(t)$ with respect to time $t$. Using Ito formula solution to Equation (5); using initial condition $N(0)=0$; as follows $[3,16]$

$$
N(t)=a\left[1-\exp \left\{-\int_{0}^{t} b(x) \mathrm{d} x-\sigma W(t)\right\}\right]
$$

For, Goel-Okumoto Model $b(x)=b$ (constant). Hence Equation (6) reduces to

$$
N(t)=a(1-e\{-(b t-\sigma W(t))\})
$$

As $N(t)$ is a random variable, its expected value will be a useful measure.

$$
E[N(t)]=a\left(1-\exp \left(-\left(b-(1 / 2) \sigma^{2}\right) t\right)\right)
$$

The Wiener process $W(t)$, is a Gaussian process and it has the following properties:

$$
\begin{aligned}
& \operatorname{Pr}[w(0)=0]=1, \\
& E[w(t)]=0, \\
& E\left[w(t) w\left(t^{\prime}\right)\right]=\min \left[t, t^{\prime}\right]
\end{aligned}
$$

\section{ANN Architecture of SRGM Based on Stochastic Differential Equation}

We can apply the neural network based approach to build a SRGM based on stochastic differential equations to predict and estimate the software reliability of software. There will be two hidden layers. Neural Network is depicted in Figure 1.

In practice, we can design different activation functions on different neurons in the hidden layer. The activation functions for the units in hidden layer in Figure $\mathbf{1}$ are defined as:

$$
\begin{aligned}
& \alpha_{1}(x)=x, \\
& \alpha_{2}(x)=\frac{x}{2} \text { and } \\
& \beta(x)=1-\mathrm{e}^{-x}
\end{aligned}
$$

The activation function for the unit in output layer is defined as

$$
\gamma(x)=x
$$

$w_{1}, w_{2}$ and $w_{3}$ are the weights of the network. Note that $w_{1}$ is the fault detection rate and $w_{2}$ is equal to $-\sigma^{2} . w_{5}$ is the proportion of total fault content in the software. We assume that there is no bias in units of hidden layers and output layer.

Input to the first unit of first hidden layer is

$$
h_{1 \_ \text {in }}(t)=w_{1} t
$$

Output from the first unit of hidden layer is

$$
h_{1}(t)=\alpha_{1}\left(h_{1 \_ \text {in }}(t)\right)=\alpha_{1}\left(w_{1} t\right)=w_{1} t
$$

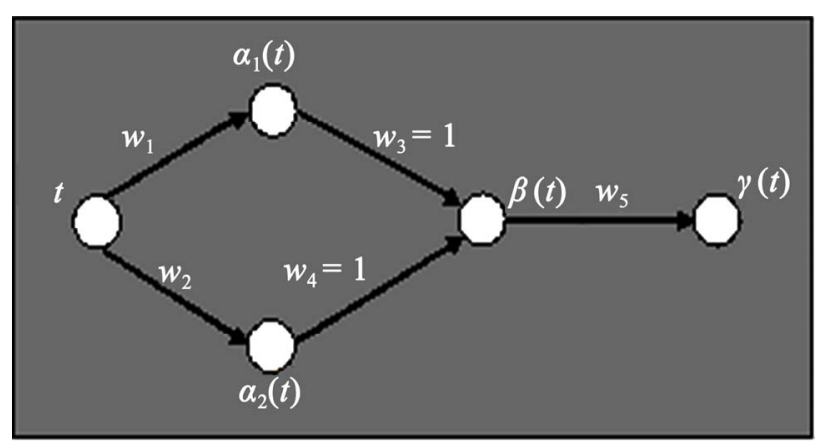

Figure 1. Network architecture of SRGM based on stochastic differential equation. 
Input to the second unit of first hidden layer is

$$
h_{2 \_ \text {in }}(t)=w_{2} t
$$

Output from the second unit of first hidden layer is

$$
h_{2}(t)=\alpha_{2}\left(h_{2-i n}(t)\right)=\alpha_{2}\left(w_{2} t\right)=\frac{w_{2} t}{2}
$$

Input to the single unit of second hidden layer is

$$
h_{3_{-} \text {in }}(t)=w_{3} w_{1} t+w_{4} \frac{w_{2} t}{2}
$$

Output from the single unit of second hidden layer is

$$
\begin{aligned}
h_{3}(t) & =\beta\left(h_{3 \_ \text {in }}(t)\right)=\beta\left(w_{3} w_{1} t+w_{4} \frac{w_{2} t}{2}\right) \\
& =1-\mathrm{e}^{-\left(w_{3} w_{1} t+w_{4} \frac{w_{2} t}{2}\right)}=1-\mathrm{e}^{-\left(w_{3} w_{1}+w_{4} \frac{w_{2}}{2}\right) t}
\end{aligned}
$$

Input to the single unit of output layer is

$$
y_{\text {in }}(t)=w_{5}\left(1-\mathrm{e}^{-\left(w_{3} w_{1}+w_{4} \frac{w_{2}}{2}\right) t}\right)
$$

Output from the single unit of output layer is

$$
\begin{aligned}
y(t) & =\gamma\left(y_{\text {in }}(t)\right)=\gamma\left(w_{5}\left(1-\mathrm{e}^{-\left(w_{3} w_{1}+w_{4} \frac{w_{2}}{2}\right) t}\right)\right) \\
& =w_{5}\left(1-\mathrm{e}^{-\left(w_{3} w_{1}+w_{4} \frac{w_{2}}{2}\right) t}\right)
\end{aligned}
$$

Using $w_{1}=b, w_{2}=-\sigma^{2}, w_{3}=1, w_{4}=1$ and $w_{5}=a$, Equation (13) is same as Equation (8), which is the mean value function for SRGM based on stochastic differential equation.

\section{Model Validation}

To assess the performance of the proposed neural-network model, we have carried out the parameter estimation on two real software failure datasets.

\subsection{Data Set 1 (DS-1)}

The first data set (DS-1) had been collected during 38 weeks and 231 faults were detected during testing. This data is cited from Misra [17].

\subsection{Data Set 2 (DS-2)}

The second data set (DS-2) had been collected during 21 weeks of testing and 26 faults were detected during testing. This data is cited from Pham [18].

\subsection{Comparison Criteria for SRGM}

The performance of SRGM are judged by their ability to fit the past software fault data (goodness-of-fit). The term goodness-of-fit denotes the question of "How good does a model fit to the data?”

1) The Mean Square Fitting Error (MSE):

The model under comparison is used to simulate the fault data, the difference between the expected values, $\hat{m}\left(t_{i}\right)$ and the observed data $y_{i}$ is measured by MSE as follows.

$$
\text { MSE }=\sum_{i=1}^{k} \frac{\left(\hat{m}\left(t_{i}\right)-y_{i}\right)^{2}}{k}
$$

where $k$ is the number of observations. The lower MSE indicates less fitting error, thus better goodness-of-fit [2].

2) Bias:

The difference between the observation and prediction of number of failures at any instant of time $i$ is known as $\mathrm{PE}_{i .}$ (prediction error). The average of PEs is known as bias. Lower the value of Bias better is the goodness of fit [19]. Prediction error is the difference between the actual and the estimated number of faults.

3) Variation:

The standard deviation of prediction error is known as variation.

$$
\text { Variation }=\sqrt{(1 / N-1) \sum\left(\mathrm{PE}_{i}-\mathrm{Bias}\right)^{2}}
$$

Lower the value of Variation better is the goodness of fit [19].

4) Root Mean Square Prediction Error:

It is a measure of closeness with which a model predicts the observation.

$$
\text { RMSPE }=\sqrt{\left(\text { Bias }^{2}+\text { Variation }^{2}\right)}
$$

Lower the value of Root Mean Square Prediction Error better is the goodness of fit [19].

\section{Data Analyses and Model Comparison}

To judge the accuracy of the proposed model (equation 13) we had used MSE, Bias, Variation and RMSPE as the performance measures. The comparison criteria results are shown in Tables $\mathbf{1}$ and 2. Goodness-of-fit curves are shown in Figures 2 and 3.

Table 1. For DS-1.

\begin{tabular}{lcccc}
\hline \multicolumn{1}{c}{ Models Compared } & MSE & Variation & Bias & RMSPE \\
\hline Goel Okumoto Model [1] & 96.848 & 6.759 & 7.236 & 9.902 \\
Proposed Model (Equation (13)) & 20.709 & 4.611 & -0.079 & 4.612 \\
\hline
\end{tabular}

Table 2. For DS-2.

\begin{tabular}{lcccc}
\hline \multicolumn{1}{c}{ Models Compared } & MSE & Variation & Bias & RMSPE \\
\hline Goel Okumoto Model [1] & 7.446 & 2.138 & 1.758 & 2.768 \\
Proposed Model (Equation (13)) & 5.721 & 2.390 & 0.530 & 2.448 \\
\hline
\end{tabular}




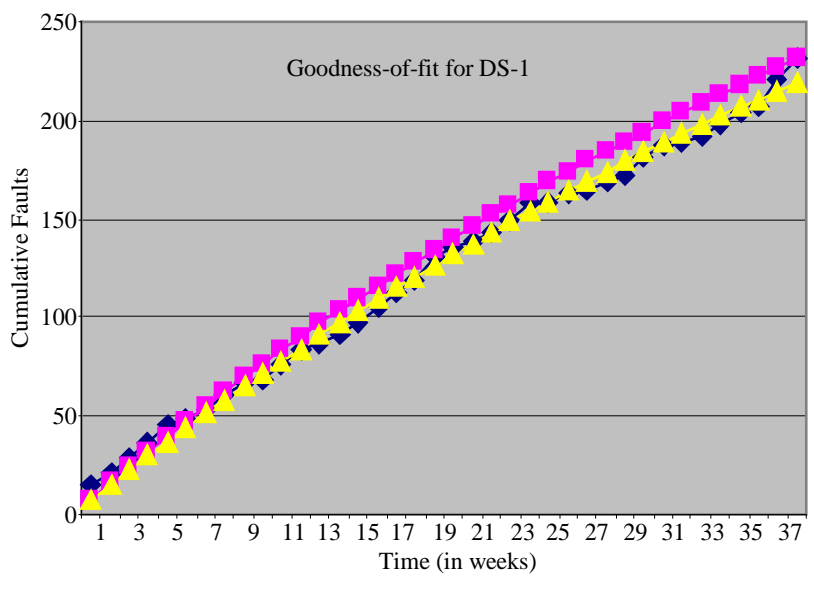

$\longrightarrow$ Actual Data $\longrightarrow$ Goel-Okumoto [1] $\longrightarrow$ Proposed Model (Equation 13)

Figure 2. Goodness of fit curve for DS-1.

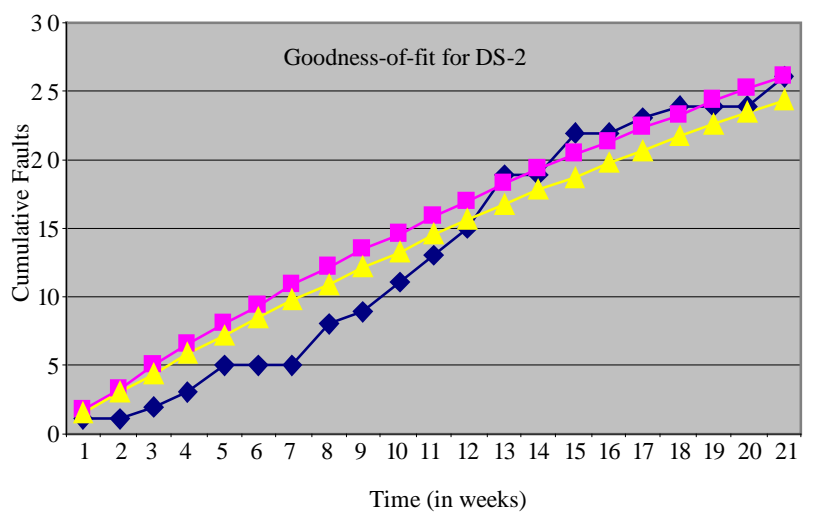

$\longrightarrow$ Actual Data $\longrightarrow$ Goel-Okumoto Model [1] $\_$Proposed Model (Equation 13)

Figure 3. Goodness of fit curve for DS-2.

\section{Conclusions}

This paper presents an SRGM based on Ito type Stochastic Differential Equations using ANN approach. The goodness of the fit analysis has been done on two real software failure datasets. The goodness-of-fit of the proposed Model is compared with Goel Okumoto model [1]. The results obtained show better fit and wider applicability of the model to different types of failure datasets. From the numerical illustrations, we see that the Proposed Model provides improved results because of lower MSE, Variation, RMSPE and Bias. The usability of SDE is not only restricted to the model described in this paper but it can also be extended to improve the results of any other SRGM. For further research the Proposed Model can be used along with error generation.

\section{REFERENCES}

[1] A. L. Goel and K. Okumoto, “Time Dependent Error De- tection Rate Model for Software Reliability and Other Performance Measure,” IEEE Transactions on Reliability, Vol. 3, 1992, pp. 206-211. doi:10.1109/TR.1979.5220566

[2] P. K. Kapur, R. B. Garg and S. Kumar, "Contributions to Hardware and Software Reliability,” World Scientific, Singapore, 1999.

[3] B. Oksendal, "Stochastic Differential Equations-An Introduction with Applications,” Springer, Berlin, 2003.

[4] S. Yamada and Y. Tamura, "A Flexible Stochastic Differential Equation Model in Distributed Development Environment," European Journal of Operational Research, Vol. 168, No. 1, 2006, pp. 143-152.

doi:10.1016/j.ejor.2004.04.034

[5] C. H. Lee, Y. T. Kim and D. H. Park, "S-Shaped Software Reliability Growth Models Derived from Stochastic Differential Equations,” IIE Transactions, Vol. 36, No. 12, 2004, pp. 1193-1199. doi:10.1080/07408170490507792

[6] N. Karunanithi and Y. K. Malaiya, "The Scaling Problem in Neural Networks for Software Reliability Prediction," Proceedings of the 3rd International IEEE Symposium of Software Reliability Engineering, Los Alamitos, 7-10 October 1992, pp. 76-82. doi:10.1109/ISSRE.1992.285856

[7] N. Karunanithi, Y. K. Malaiya and D. Whitley, "Prediction of Software Reliability Using Neural Networks," Proceedings of the 2nd IEEE International Symposium on Software Reliability Engineering, Los Alamitos, 17-18 May 1991, pp. 124-130.

[8] N. Karunanithi, D. Whitley and Y. K. Malaiya, "Using Neural Networks in Reliability Prediction,” IEEE Software, Vol. 9, No. 4, 1992, pp. 53-59. doi:10.1109/52.143107

[9] K. Y. Cai, L. Cai, W. D. Wang, Z. Y. Yu and D. Zhang, "On the Neural Network Approach in Software Reliability Modeling,” The Journal of Systems and Software, Vol. 58, No. 1, 2001, pp. 47-62. doi:10.1016/S0164-1212(01)00027-9

[10] S. A. Sherer, "Software Fault Prediction," Journal of Systems and Software, Vol. 29, No. 2, 1995, pp. 97-105. doi:10.1016/0164-1212(94)00051-N

[11] T. M. Khoshgoftar and R. M. Szabo, "Using Neural Networks to Predict Software Faults during Testing," IEEE Transactions on Reliability, Vol. 45, No. 3, 1996, pp. 456462. doi:10.1109/24.537016

[12] Y. S. Su, C. Y. Huang and Y. S. Chen, “An Artificial Neural-Network Based Approach to Software Reliability Assessment," Proceedings of IEEE Region 10 Conference, Melbourne, 21-24 November 2005, pp. 1-6.

[13] P. K. Kapur, S. K. Khatri, M. Basirzadeh and N. Dembla, "Modeling Software Reliability Growth in Distributed Environment Using Artificial Neural-Networks,” In: S. K. Khatri and B. Kumar, Eds., Proceedings of International Conference on Reliability, Infocom Technology and Optimization, Faridabad, 1-3 November 2010, pp. 372-382.

[14] P. K. Kapur, S. K. Khatri and D. N. Goswami, “A Generalized Dynamic Integrated Software Reliability Growth Model Based on Neural-Network Approach,” Proceed- 
ings of International Conference on Reliability, Safety and Quality Engineering, 5-7 January 2008, pp. 831-838.

[15] S. K. Khatri, R. Kapur, P. Johri and P. Sharma, “Artificial Neural-Networks Based Software Reliability Growth Modeling with Two types of Imperfect Debugging,” In: S. K. Khatri and B. Kumar, Eds., Proceedings of International Conference on Reliability, Infocom Technology and Optimization, Faridabad, 1-3 November 2010, pp. 122-133.

[16] S. Yamada, A. Nishigaki and M. Kimura, "A Stochastic Differential Equation Model for Software Reliability Assessment and Its Goodness of Fit,” International Journal of Reliability and Applications, Vol. 4, No. 1, 2003, pp. 1-11.

[17] P. N. Misra, "Software Reliability Analysis,” IBM System Journal, Vol. 22, No. 3, 1983, pp. 262-270. doi:10.1147/sj.223.0262

[18] H. Pham, "Software Reliability," Springer-Verlag, Singapore City, 2000.

[19] K. Pillai and V. S. S. Nair, "A Model for Software Development effort and Cost Estimation," IEEE Transactions on Software Engineering, Vol. 23, No. 8, 1997, pp. 485-497. doi:10.1109/32.624305 